\title{
Segmental arterial mediolysis, a vasculitis mimic: Case report and review of literature
}

\author{
Neha Garg ${ }^{1}$, Frederick S. Keller ${ }^{2}$ \\ 1. Arthritis \& Rheumatic Diseases, Oregon Health \& Science University, Portland, United States. 2. Dotter Interventional \\ Institute, Oregon Health \& Sciences University, Portland, United States.
}

Correspondence: Neha Garg. Address: Arthritis \& Rheumatic Diseases, Oregon Health \& Science University, 3181 SW Sam Jackson Park Road, Mail Code OP-09, Portland, OR 97239, United States. Email: docnehagarg@gmail.com

Received: March 6, 2014

Accepted: June 4, 2014

Online Published: June 12, 2014

DOI : $10.5430 /$ ijdi.v1n2p101

URL: http://dx.doi.org/10.5430/ijdi.v1n2p101

\begin{abstract}
Segmental Arterial Mediolysis (SAM) is a non-inflammatory vascular condition that most commonly affects the medium sized vessels in the abdomen including splanchanic and renal arteries. Its angiographic appearance closely mimics other conditions affecting these blood vessels, such as polyarteritis nodosa (PAN), infections such as syphilis and fibromuscular dysplasia. SAM is under-recognized, however it is important to distinguish it from PAN because the management of the two conditions differs significantly. Here we report a case of a woman presenting with intra-abdominal bleeding, with imaging evidence for large retroperitoneal hematoma. Arteriography revealed diffuse, segmental dilation and aneurysms of the arterial supply to the entire colon. Discussion includes review of current understanding of physiopathology, clinical features, imaging, and differential diagnosis of this rare disease. Recent highlights on the disease course are also discussed.
\end{abstract}

\section{Keywords}

Segmental arterial mediolysis, Arteriopathy, Vasculitis, Polyarteritis nodosa, Fibromuscular dysplasia

\section{I ntroduction}

Segmental arterial mediolysis (SAM) is a rare idiopathic non-arteriosclerotic and non-inflammatory vascular disease of medium sized blood vessels. It mostly involves the abdominal splanchnic arteries and is known to produce spontaneous hemorrhage into the mesentery. It is important to distinguish SAM from conditions like Polyarteritis Nodosa (PAN), Takayasu's arteritis, Henoch-Schonlein Purpura, Behcet’s disease, fibromuscular dysplasia (FMD), mycotic aneurysms and congenital vascular disorders. SAM was first described by Slavin ${ }^{[1]}$, and has been reported under different names for example "segmental mediolytic arteriopathy" ${ }^{[2]}$. SAM has an angiographic appearance that close mimics that of medium vessel vasculitides such as PAN making it extremely important to recognize and distinguish it from inflammatory disorders since the current management of the two conditions differ significantly.

\section{Case presentation}

A 56-year-old female was evaluated for acute onset severe abdominal pain, vomiting, diarrhea, headache, syncope and significant drop in hematocrit. She had a past medical history of hypertension, hypothyroidism, and chronic spinal pain. 
Her medications included metoprolol, ibuprofen and oxycodone as needed for pain. She had a remote history of hysterectomy, cholecystectomy, appendectomy, and spinal fusion. Social history was significant for a fifteen-pack year smoking history. On presentation to an outside hospital, she was found to be hypotensive and tachycardic with a blood pressure of 103/77, heart rate of 117. She was afebrile and had normal oxygen saturation. Physical examination was remarkable for diffuse tenderness on abdominal palpation along with rebound and guarding. Her initial hematocrit (Hct) was $39.5 \%$, which dropped to $33 \%$ in four hours, and White blood cell count (WBC) was $26.8 \mathrm{~K} / \mathrm{cu} \mathrm{mm}$. A Computed Tomography (CT) scan showed a large fluid collection concerning for hemoperitoneum within the lesser sac. She was treated with continuous intra-venous fluids and transferred to our hospital. Her Hct at our hospital was $27 \%$ and WBC was $19.9 \mathrm{~K} / \mathrm{cu} \mathrm{mm}$. A dedicated CT angiogram of the abdomen and pelvis revealed a large evolving left upper retroperitoneal and small bowel mesenteric hematoma tracking into the pancreatiocolic ligament towards the splenic flexure of the colon and extending into the left anterior pararenal space. Vasculature was notable for multiple small sub-centimeter visceral arterial aneurysms, including a 7-mm inferior mesenteric artery (IMA)-left colic branch aneurysm, distal gastro-duodenal artery (GDA), proximal superior mesenteric artery (SMA) branch aneurysms, and aneurysms in the posterior splenic hilum (see Figure 1). These findings were initially reported as most suggestive of visceral vasculitis such as PAN. Further laboratory evaluation revealed a normal erythrocyte sedimentation rate, C-reactive protein, urine analysis, negative anti-nuclear antibody, negative anti-neutrophilic cytoplasmic antibodies and normal complements. Infectious work up was negative for acute or chronic viral infections including hepatitis B, hepatitis C, HIV and syphilis. Given the history of headache, Magnetic Resonance Imaging (MRI) and MR Angiogram of the brain were done, which revealed no abnormality.

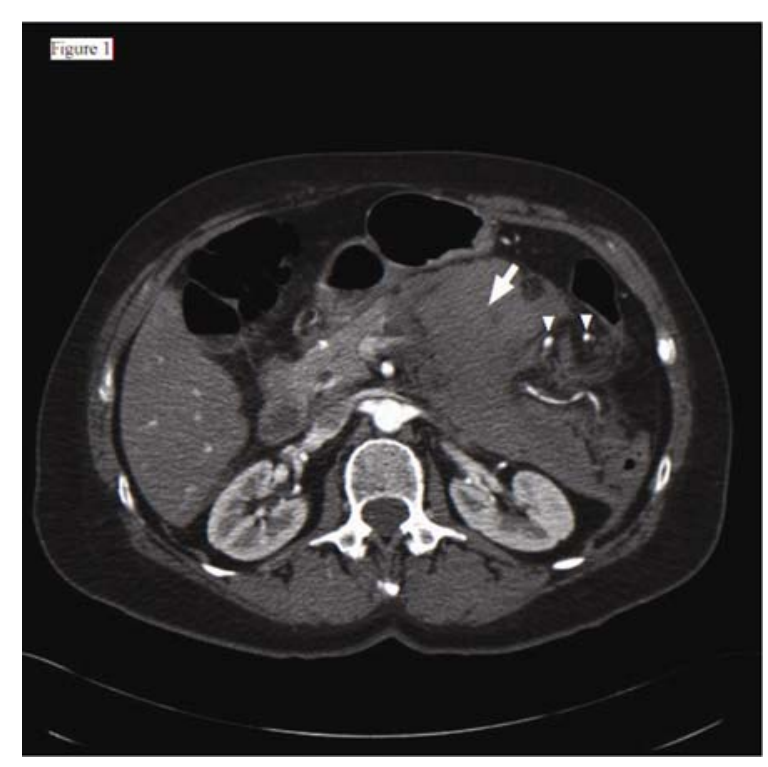

Figure 1. It shows Abdominal CT demonstrating a large retroperitoneal and mesenteric hematoma (arrow) containing multiple small visceral arterial aneurysms within it (arrowheads).

On further questioning, the patient denied having constitutional symptoms, cutaneous lesions, post-prandial abdominal pain, or a history of uncontrolled hypertension. A visceral digital subtraction angiogram revealed diffuse, segmental dilation and aneurysms of the arterial supply to the entire colon (see Figure 2, 3). The inferior mesenteric arteriogram showed abnormal dilatations of left colic and the marginal arteries. The superior mesenteric arteriogram showed abnormal dilatations of middle colic artery. Similarly, celiac arteriogram demonstrated a small aneurysm of the distal splenic artery. Arterial branches to the small bowel and renal arteriograms were normal.

With these angiographic findings, her presentation was deemed more consistent with SAM. Lack of systemic prodromal symptoms of fever or malaise, lack of cutaneous or renal involvement, and normal inflammatory markers was thought to 
be atypical for vasculitis. The patient was treated conservatively and was discharged after resolution of abdominal pain and stabilization of hematocrit without needing any radiological or surgical intervention. She was asymptomatic on follow up 6 months later and declined a repeat angiogram.

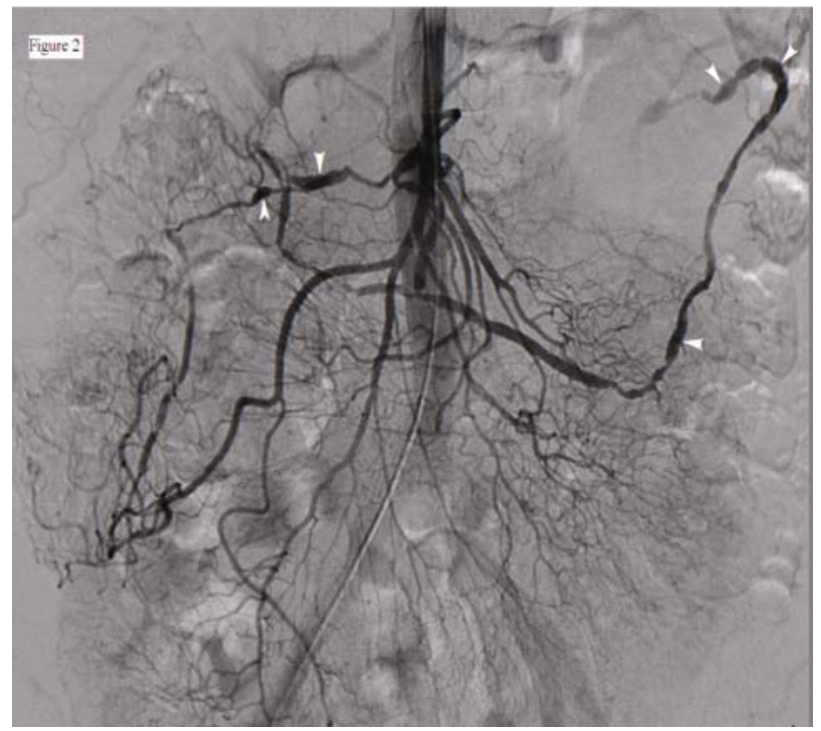

Figure 2. It shows Superior mesenteric arteriogram with fusiform aneurysms (arrowheads) in branches of the middle colic artery. Avascular area in the distal transverse colon and splenic flexure represents a hematoma.

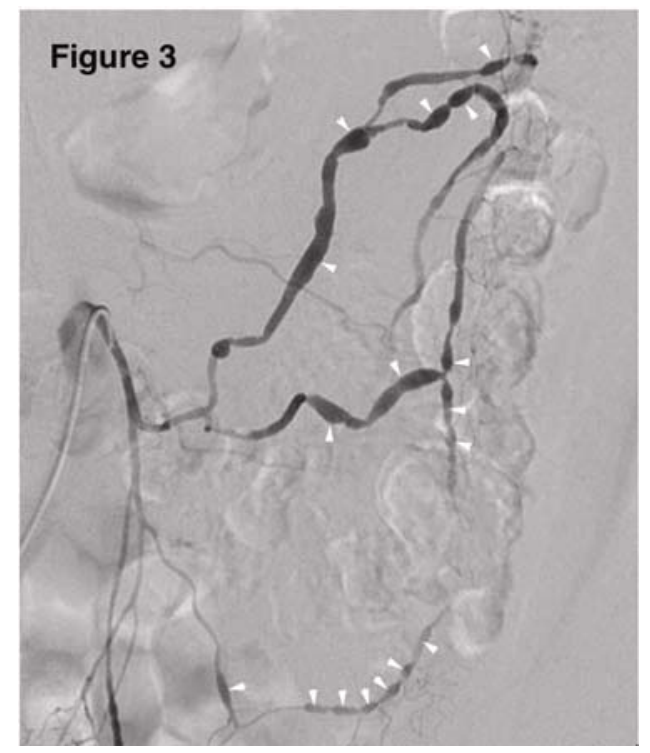

Figure 3. It shows Inferior mesenteric arteriogram demonstrating multiple fusiform aneurysms (arrowheads) in the left colic artery and its branches.

\section{Discussion}

SAM is a rare non-atherosclerotic non-inflammatory arteriopathy of unknown etiology characterized by lysis of medial layer of arterial wall resulting in stenosis, occlusion, aneurysmal formation and dissection ${ }^{[3]}$. Diagnosis remains clinical, 
aided by imaging, most commonly CT and conventional angiography. Histology remains the gold standard of diagnosis, however is rarely available ante-mortem.

The incidence and prevalence of SAM is not known and is likely under-estimated because many cases are asymptomatic, and a detailed vascular work up is not part of routine evaluation of abdominal pain in most settings. Gender predilection is variable in different reports with greater prevalence in older women in some reports vs. slight male preponderance in others ${ }^{[3]}$. Mean age is usually reported to be around 60 years ${ }^{[3]}$, but neonatal involvement has been reported ${ }^{[4]}$. No clear disease associations with hypertension or smoking, and no familial or genetic predispositions have been described.

The most common presenting symptom is abdominal pain, occurring in about two-thirds of patients. About a third can present with hemodynamic shock (from life threatening hemorrhage) and 14\% may present with neurologic symptoms according a recent systematic review ${ }^{[3]}$. But the true incidence of life threatening hemorrhage in SAM remains unclear since many cases are asymptomatic. Virtually all cases reported have symptoms pertaining to either visceral or cerebral vascular bed involvement. Intra-abdominal vasculature is most commonly involved including mesenteric, gastric, splenic, and pancreatic arteries ${ }^{[3]}$. Involvement of renal, coronary and cranial vessels has been reported as well ${ }^{[5-9]}$.

PAN and FMD may be the closest differential diagnoses of SAM. Congenital vascular conditions including Neurofibromatosis type 1 (usually causing coarctation of aorta and renal artery stenosis) and Ehlers-Danlos Syndrome Type IV, and infectious causes of arterial aneurysms including fungal infections and syphilis should also be considered in the differential diagnosis. PAN commonly presents with involvement of splanchnic (usually renal) and skin vasculature and presents with cutaneous nodules or ulcers, hypertension, along with constitutional symptoms of fever and weight loss with high inflammatory markers. The angiographic appearance closely resembles that of SAM with 'beading' of vessel walls. The histopathological hallmark of PAN is presence of inflammatory infiltrate within the vessel wall along with fibrinoid necrosis of the vessel wall, which is absent in SAM. SAM may be very difficult to distinguish from FMD especially the subclinical form. Indeed, SAM has been purported to be a variant of FMD ${ }^{[10-14]}$.

Little is known about the etiopathogenesis of SAM. It is considered a non-arteriosclerotic and non-inflammatory disorder of arteries ${ }^{[15]}$. Characteristic histologic lesions consist of necrosis and vacuolar degeneration of the outer layer of arterial media (the injurious phase), followed by a tear separating the outer medial muscle from the adventitia (the reparative phase) of SAM that are distributed usually in a segmental distribution. These lesions then cause dilatation, aneurysms, and thrombi causing stenosis and occlusions.

Digital subtraction imaging (DSA) is the most commonly used diagnostic test. The characteristic pattern is of focal aneurysms and beading, notably with an otherwise normal vascular appearance ${ }^{[11]}$. Six angiographic patterns have been identified: (a) arterial dilatation, (b) single aneurysm, (c) multiple aneurysms, (d) dissecting hematomas, (e) arterial stenosis, and (f) arterial occlusions by thromboses ${ }^{[10]}$. The aneurysms are mostly multiple, with characteristic string-of-beads appearance. Skip lesions can occur without any predilection for sites of bifurcations, unlike arteriosclerosis. Noninvasive methods such as CT angiography have shown good correlation with conventional angiograms and may have great value in making initial diagnosis and in follow up care ${ }^{[15]}$.

Mortality is usually caused by a life-threatening hemorrhage and can be as high as $50 \%$ in earlier reports, and has dropped to about $25 \%$ with recent advances in diagnostic and therapeutic techniques ${ }^{[3,16]}$. Most deaths occur before or soon after presentation to a hospital ${ }^{[3]}$. Many recent reports have had successful outcomes with endovascular, or open surgical management. Initial management for life threatening hemorrhage remains emergency exploratory laparotomy with ligation of bleeding vessels and/or resection of aneurysms. Endovascular repairs for SAM related aneurysms have a success rate approaching $90 \%$ with very low morbidity ${ }^{[3,17]}$. Open surgery as the primary management or after failed endovascular intervention also had a mortality rate of $9 \%{ }^{[3]}$. Severe hemorrhage may necessitate end-organ resection (i.e., partial colectomy, nephrectomy, or splenectomy) secondary to ischemia or hemorrhage. Endovascular stenting or surgical bypass is needed for stenotic arterial segments. 
The natural course of disease remains unpredictable. Over time, the smaller aneurysms may decrease in size, and may eventually resolve. However, more commonly vascular abnormalities persist, and usually have an asymptomatic course, but less commonly may have dissections and hemorrhage later in life ${ }^{[10,15]}$. In stable patients, there have been reports of successful management with conservative or medical therapy with aspirin and anti-hypertensive medicines, although the efficacy of such medical therapies is not clear. Elective treatment of asymptomatic aneurysms remains controversial and no clear predictable algorithms exist to gauge the risk of an aneurysmal rupture in future.

In summary, radiologists should have a high index of suspicion for SAM since they are best suited to make the diagnosis in most cases, potentially treat it with interventional techniques and safely follow these patients.

\section{References}

[1] Slavin RE, Gonzalez-Vitale JC, Segmental M. Arteritis: a clinical pathologic study. Lab Invest. 1976; 35: 23 e9.

[2] Hagspiel KD, Angle JF, Spinosa DJ, Matsumoto AH. Diagnosis please. Case 13: polyarteritis nodosa--systemic necrotizing vasculitis with involvement of hepatic and superior mesenteric arteries. Radiology. 1999 Aug; 212(2): 359-64. http://dx.doi.org/10.1148/radiology.212.2.r99au38359

[3] Michael Shenouda, Celia Riga, Yaser Naji, and Sophie Renton. Segmental Arterial Mediolysis: A Systematic Review of 85 Cases. Ann Vasc Surg. 2014; 28: 269-277. http://dx.doi.org/10.1016/j.avsg.2013.03.003

[4] Eifinger F, Fries J, Bald R, Körber F, Kribs A, Roth B. Segmental arterial mediolysis in a preterm. J Perinatol. 2004 Jul; 24(7): 461-4. http://dx.doi.org/10.1038/sj.jp.7211130

[5] Horsley-Silva JL, Ngamruenphong S, Frey GT, Paz-Fumagalli R, Lewis MD. Segmental arterial mediolysis: a case of mistaken hemorrhagic pancreatitis and review of the literature.JOP. 2014 Jan 10; 15(1): 72-7.

[6] Tabassum A, Sasani S, Majid AJ, Henderson C, Merrett ND. Segmental arterial mediolysis of left gastric artery: a case report and review of pathology. BMC Clin Pathol. 2013 Oct 29; 13(1): 26. http://dx.doi.org/10.1186/1472-6890-13-26

[7] Alturkustani M, Ang LC.Intracranial segmental arterial mediolysis: report of 2 cases and review of the literature. Am J Forensic Med Pathol. 2013 Jun; 34(2): 98-102. http://dx.doi.org/10.1097/PAF.0b013e31828879e8

[8] Oki T, Adachi H, Tahara H, Kino S. Spontaneous renal artery dissection with renal infarction: a case report. Hinyokika Kiyo. 2011 Nov; 57(11): 611-4.

[9] Soulen MC, Cohen DL, Itkin M, Townsend RR, Roberts DA. Segmental arterial mediolysis: angioplasty of bilateral renal artery stenoses with 2-year imaging follow-up. J Vasc Interv Radiol. 2004 Jul; 15(7): 763-7. http://dx.doi.org/10.1097/01.RVI.0000133543.32123.DC

[10] Richard E. Slavin. Segmental arterial mediolysis: course, sequelae, prognosis, and pathologic-radiologic correlation. Cardiovascular Pathology. 2009; 18: 352-360. http://dx.doi.org/10.1016/j.carpath.2008.09.001

[11] Heritz DM, Butany J, Johnston KW, Sniderman KW. Intraabdominal hemorrhage as a result of segmental mediolytic arteritis of an omental artery: case report. J Vasc Surg. 1990; 12:561-565. http://dx.doi.org/10.1016/0741-5214(90)90011-X

[12] Slavin RE, Saeki K, Bhagavan B, Maas AE. Segmental arterial mediolysis: a precursor to fibromuscular dysplasia? Mod Pathol. 1995; 8:287-94.

[13] Chan RJ, Goodman TA, Aretz TH, Lie JT. Segmen- tal mediolytic arteriopathy of the splenic and he- patic arteries mimicking systemic necrotizing vas- culitis. Arthritis Rheum. 1998; 41:935-938. http://dx.doi.org/10.1002/1529-0131(199805)41:5<935::AID-ART22>3.0.CO;2-N

[14] Slavin RE, Cafferty L, Cartwright J Jr. Segmental mediolytic arteritis. A clinicopathologic and ultrastructural study of two cases. Am J Surg Pathol. 1989; 13: 558e68.

[15] Michael M, Widmer U, Wildermuth S, Barghorn A, Duewell S, Pfammatter T. Segmental arterial mediolysis: CTA findings at presentation and follow-up. AJR Am J Roentgenol. 2006; 187: 1463-9. http://dx.doi.org/10.2214/AJR.05.0281

[16] Sakano T, Morita K, Imaki M, et al. Segmental arterial mediolysis studied by repeated angiography. Br J Radiol. $1997 ;$ 70: 656e8.

[17] Ryan JM, Suhocki PV, Smith TP. Coil embolization of segmental arterial mediolysis of the hepatic artery. J Vasc Interv Radiol. 2000; 11: 865e8. 